\title{
ON THE MOMENT PROBLEM FOR A FINITE INTERVAL
}

\section{BY T. H. HILDEBRANDT}

Hausdorff* and Schoenberg $\dagger$ have shown that a necessary and sufficient condition that there exist a function of bounded variation $\chi(t)$, such that we have, for every $n, \mu_{n}=\int_{0}^{1} t^{n} d \chi(t)$, is that $\sum_{m=0}^{n}\left(\begin{array}{l}m \\ n\end{array}\right)\left|\Delta^{n-m} \mu_{m}\right|$ form a bounded sequence. The fact that if such a function $\chi(t)$ exists, then $\Delta^{m} \mu_{n}=\int_{0}^{1} t^{n}(1-t)^{m} d \chi(t)$, suggests that perhaps the sufficiency part of this theorem can be easily demonstrated by using the Bernstein polynomials and the Riesz theorem on linear functional operations on the set of continuous functions.

For any function $f(t)$ on $(0,1)$ the $n$th Bernstein polynomial is defined by the formula

$$
B_{n}(f)=\sum_{m=0}^{n}\left(\begin{array}{l}
n \\
m
\end{array}\right) f(m / n) t^{m}(1-t)^{n-m},
$$

and it is well known that the polynomials $B_{n}(f)$ converge uniformly to $f(t)$ if $f$ is continuous. Further, it can be easily shown by induction $\ddagger$ that if $P_{k}(t)$ is any polynomial of degree $k$, then

$$
B_{n}\left(P_{k}\right)=P_{k}(t)+\sum_{i=1}^{k-1} A_{i k}(t) / n^{i}
$$

where $A_{i k}(t)$ are polynomials of degree at most $k$ and are independent of $n$.

Define now a linear operation $U$ on polynomials $P_{k}(t)$ by the condition

$$
U\left(P_{k}\right)=U\left(a_{0}+a_{1} t+\cdots+a_{k} t^{k}\right)=\sum_{i=0}^{k} a_{i} \mu_{i} .
$$

* Mathematische Zeitschrift, vol. 16 (1923), p. 238.

$\dagger$ This Bulletin, vol. 38 (1932), p. 72.

¥ Following the suggestion in Pólya and Szegö, Aufgaben und Lehrsätze aus der Analysis, vol. I, p. 6, Ex. 40; and p. 66, Ex. 144. 
Then for any function $f$,

$$
U\left(B_{n}(f)\right)=\sum_{m=0}^{n} f(m / n)\left(\begin{array}{l}
n \\
m
\end{array}\right) \Delta^{n-m} \mu_{m} .
$$

As a consequence, for every $n$,

$$
\left|U\left(B_{n}(f)\right)\right| \leqq F \cdot M
$$

where $F$ is the least upper bound of $|f(t)|$ on $(0,1)$ and $M$ is the least upper bound of $\sum_{m=0}^{n}\left(\begin{array}{l}n \\ m\end{array}\right)\left|\Delta^{n-m} \mu_{m}\right|$. Hence if $P_{k}$ is any polynomial of degree $k$, then

so that

$$
\left|U\left(P_{k}\right)\right| \leqq\left|U\left(B_{n}\left(P_{k}\right)\right)\right|+\sum_{i=0}^{k-1}\left|U\left(A_{i k}\right)\right| / n^{i}
$$

$$
\left|U\left(P_{k}\right)\right| \leqq \max \left|P_{k}\right| \cdot M .
$$

It follows from this inequality that if $f$ is any continuous function on $(0,1)$, then the sequence of numbers $U\left(B_{n}(f)\right)$ satisfy the Cauchy condition of convergence, that is, converge to a finite limit, which limit we denote by $U(f)$. Obviously $U(f)$ is linear in $f$ and $|U(f)| \leqq F \cdot M$. Hence by the Riesz theorem on linear limited operations, there exists a function $\chi(t)$ of bounded variation, such that

$$
U(f)=\int_{0}^{1} f(t) d \chi(t), \text { that is, } \mu_{n}=\int_{0}^{1} t^{n} d \chi(t) .
$$

The University of Michigan 